\title{
Sorbitol accumulation is altered in Type 1 (insulin-dependent) diabetes mellitus
}

\author{
J. I. Malone ${ }^{1,2}$, G. Knox ${ }^{2}$ and C. Harvey ${ }^{2}$ \\ ${ }^{1}$ USF Diabetes Center and ${ }^{2}$ Department of Pediatrics, College of Medicine, University of South Florida, Tampa, Florida, USA
}

\begin{abstract}
Summary. Intracellular sorbitol accumulation has been implicated as an aetiological factor for many of the complications of diabetes mellitus. Erythrocyte sorbitol is found in higher concentration in Type 1 (insulin-dependent) diabetic patients than in normal subjects. When sorbitol accumulation is corrected for its immediate prescursor glucose (sorbitol/glucose ratio), the polyol accumulation remains significantly greater in erythrocytes from the Type 1 diabetic. Erythrocytes from Type 1 diabetic patients exposed to normal extracellular glucose concentrations for $3 \mathrm{~h}$ in vitro, accumulate more sorbitol and fructose than normal cells in the same incubation system. Near-normalization of plasma glucose in Type 1 diabetic pat-
\end{abstract}

ients for $12 \mathrm{~h}$ did not result in normal erythrocyte sorbitol levels. The increased sorbitol accumulation in erythrocytes from Type 1 diabetic subjects may reflect similar activity in the lens and nerve in long-standing diabetes. This increased sorbitol production was found in most, but not all, individuals with diabetes and appears to be an acquired characteristic. Those factors influencing enhanced tissue sorbitol accumulation may be important in the aetiology of diabetes-associated complications.

Key words: Type 1 diabetes, sorbitol, fructose, polyol pathway.
Intracellular sorbitol accumulation has been reported to be an aetiological factor in cataract development [1], peripheral neuropathy [2], and possibly the vascular complications of diabetes mellitus [3]. We have previously demonstrated that sorbitol content inside the erythrocytes of Type 1 (insulin-dependent) diabetic patients is greater than in those from normal subjects [4]. That study demonstrated that erytrocyte sorbitol concentrations correlate well with the coincident plasma glucose levels. Sorbitol accumulation in the intact erythrocyte responds to a spectrum of glucose concentrations in a fashion consistent with the activity of aldose reductase and the polyol pathway. Gabbay and Cathcart [5] indicated that aldose reductase is not found in human erythrocytes. Beutler and Guinto [6], however, reported aldose-reducing activity in erythrocytes with kinetic characteristics similar to aldose reductase activity identified in the human aorta [7]. Crabbe and Halder [8] isolated a different aldose-reducing activity in human erythrocytes with kinetic properties similar to calf lens aldose reductase. Although isolation and characterization of aldose reductase in the erythrocytes, lens, and nerves of a single species have not been achieved to date, there is evidence that sorbitol accumulation in the erythrocyte does reflect coincident sorbitol levels in the lens and peripheral nerve [9].
The purpose of this study was to determine whether the elevated sorbitol level in the erythrocyte is merely a reflection of the coincident plasma glucose concentration or an indicator of altered metabolic activity related to diabetes mellitus.

\section{Subjects and methods}

\section{Subjects}

Blood was collected after an $8 \mathrm{~h}$ fast from 140 Type 1 diabetic patients (mean age $17.2 \pm 0.43$ years, range $8-21$ years) and 50 normal subjects of similar ages. Duration of diabetes was $9.4 \pm 0.48$ years (5-14 years) in this group which had no clinical evidence of diabetic complications. Four members of this group (two females, two males, aged 16-18 years with duration of diabetes $7-10$ years) had their plasma glucose maintained at a level $<8.33 \mathrm{mmol} / 1$ for $12-14 \mathrm{~h}$ by continuous intravenous crystalline insulin administration during hospitalization to improve poor metabolic control. Blood was collected at hourly intervals from these subjects as well as one normal female ( 22 years) and one normal male ( 28 years). Erythrocytes were separated immediately from the plasma and prepared for analysis as described previously [4].

\section{Methods}

Erythrocyte sorbitol was measured by a coupled fluorometric enzyme assay [4]. Plasma and extracellular glucose were measured by a glucose oxidase method using a glucose analyzer (Beckman, Fullerton, 
Table 1. Erythrocyte sorbitol and extracellular glucose in Type 1 diabetic and normal subjects

\begin{tabular}{llll}
\hline & $\begin{array}{l}\text { Normal } \\
\text { subjects } \\
(n=50)\end{array}$ & $\begin{array}{l}\text { Type 1 } \\
\text { diabetic patients } \\
(n=140)\end{array}$ & $p$ \\
\hline $\begin{array}{l}\text { Erythrocyte } \\
\text { sorbitol } \\
\text { (nmol/g } \\
\text { haemoglobin) }\end{array}$ & $10.3 \pm 0.8$ & $36.6 \pm 1.7$ & $<0.0001$ \\
$\begin{array}{l}\text { Sorbitol/glucose } \\
\text { ratio }\end{array}$ & $12.1 \pm 1.0$ & $20.6 \pm 0.9$ & $<0.0001$ \\
$\begin{array}{l}\text { HbA } \\
\text { ic }(\%)\end{array}$ & $4.4 \pm 0.3$ & $10.8 \pm 0.3$ & $<0.0001$ \\
\hline
\end{tabular}

Results expressed as mean $\pm \mathrm{SEM}$

California, USA). Fructose was measured by gas liquid chromatography as a trimethylsilyl ether derivative prepared by the method of Sweeley et al. [10]. Erythrocytes were incubated with glucose oxidase to remove glucose [4] and were chromatographed isothermally at $200^{\circ} \mathrm{C}$ using a gas chromatograph (Miodel 900, Perkin-Elmer, Norwalk, Connecticut, USA) equipped with a flame ionization detector and a $1.83-\mathrm{m}$ column ( 6 feet) containing JXR $3 \%$ on $100 / 120$ Gas Chrom Q (Applied Science Laboratories, State College, Penn, USA).

Since erythrocyte sorbitol levels have been shown to correlate with coincident plasma glucose concentration $[4,11]$, it was decided to express sorbitol levels as a function of the coincident plasma glucose concentration. This expression is the sorbitol/glucose ratio. This manipulation corrects the intracellular sorbitol concentration for the influence of variable plasma glucose levels common to Type 1 diabetes. The sorbitol/glucose ratio allows direct comparison of the sorbitol levels in normal cells and in cells from Type 1 diabetic patients to determine whether the reported difference in sorbitol accumulation is merely a reflection or coincident plasma glucose concentration.

Incubation studies of erythrocytes were carried out to compare sorbitol accumulation in normal and Type 1 diabetic subjects exposed to identical glucose concentrations. Heparinized samples of blood were collected from 10 normal and 11 Type 1 diabetic individuals and centrifuged at $600 \mathrm{~g}$ for $5 \mathrm{~min}$ at $4{ }^{\circ} \mathrm{C}$; the white cells, platelets and plasma were removed. The erythrocytes were then suspended in $\mathrm{NaCl}$ $(0.154 \mathrm{~mol} / 1)$ and centrifuged at $2000 \mathrm{~g}$ for $10 \mathrm{~min}$. This washing procedure was repeated three times. Washed erythrocytes $(3 \mathrm{ml})$ were suspended in Krebs buffer ( $\mathrm{pH} 7.4$ ) to a final volume of $10 \mathrm{ml}$. Replicate samples from each subject were incubated for $3 \mathrm{~h}$ at $37^{\circ} \mathrm{C}$ in Krebs bicarbonate buffer containing an initial glucose concentration of either $5.6,16.7$ or $27.8 \mathrm{mmol} / 1$ as described previously [4]. The reduction in media glucose per $g$ haemoglobin at the end of each incubation period was used as an indicator of glucose utilization and erythrocyte viability [11]. The final erythrocyte suspension contained $<100$ leucocytes $/ \mathrm{mm}^{3}$ as measured by a HC337 haematology analyzer (Hycel, Houston, Texas, USA). This degree of contamination did not contribute significantly to the sorbitol and fructose levels measured in these studies [12].

Haemoglobin $\mathrm{A}_{1 \mathrm{C}}\left(\mathrm{HbA}_{1 c}\right)$ was measured by high performance liquid chromatography with a modification of the method of Cole et al. [13]. Washed erythrocytes were incubated in three volumes of $\mathrm{NaCl}$ for $24 \mathrm{~h}$ at $22^{\circ} \mathrm{C}$ to remove the labile fraction. The column temperature was maintained at $16^{\circ} \mathrm{C}$ and the peak areas were integrated and rendered normal by a data reduction system (Perkin-Elmer, Norwalk, Connecticut, USA).

\section{Statistical analysis}

All data are expressed as mean \pm SEM and statistical analysis was carried out by the two-tailed Student's t-test.

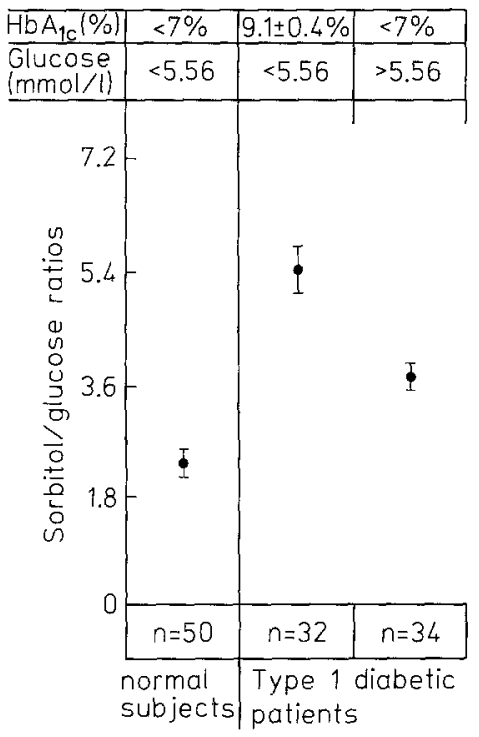

Fig. 1. Comparison of sorbitol/glucose ratios in erythrocytes collected from 50 non-diabetic and 66 Type 1 diabetic individuals (mean \pm SEM). The mean sorbitol/glucose ratio for cells collected from Type 1 diabetic patients when the plasma glucose was in the normal range $(<5.6 \mathrm{mmol} / 1)$ is significantly greater than the normal sorbitol/glucose ratio $(p<0.001)$. The mean sorbitol/glucose ratio in cells from Type 1 diabetic patients with $\mathrm{HbA}_{1 c}$ values in the normal range $(<7 \%)$ is significantly greater than the sorbitol/glucose ratio found in normal cells $(p<0.001)$. The mean plasma glucose for the Type 1 diabetic patients at the time of collection was $8.4 \pm 0.9 \mathrm{mmol} / 1$

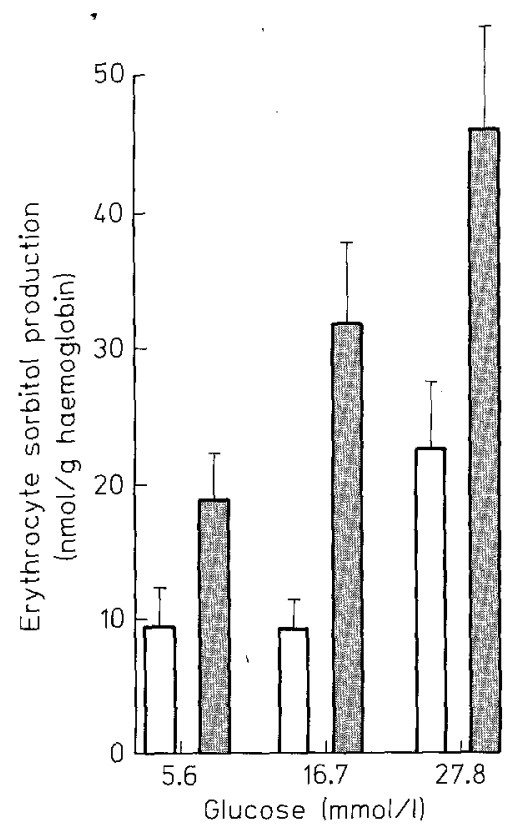

Fig. 2. Sorbitol accumulation in erythrocytes (mean \pm SEM) incubated for $3 \mathrm{~h}$ at the glucose concentrations $(5.6,16.7$ or $27.8 \mathrm{mmol} / 1)$. The erythrocyte sorbitol concentration at the end of the first hour of incubation was considered the base line concentration. Type 1 diabetes

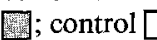

\section{Results}

Mean erythrocyte sorbitol, sorbitol/glucose ratios and $\mathrm{HbA}_{1 \mathrm{c}}$ values for erythrocytes from Type 1 diabetic and normal subjects are shown in Table 1 . The mean values 


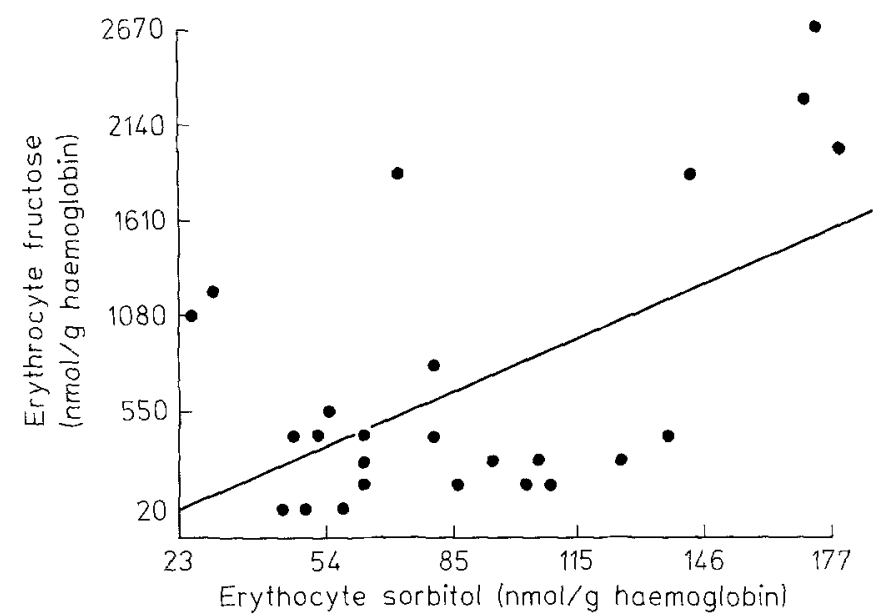

Fig. 3. Coincident erythrocyte sorbitol and fructose measured at the conclusion of the 3 - $h$ incubation period shown in Figure 2 . The 25 fructose values measured were the only ones of the 63 incubations with fructose levels within the detection limits of the assay. All fructose was found in erythrocytes from Type 1 diabetic patients. The linear regression line: $r=0.562, p<0.0037$

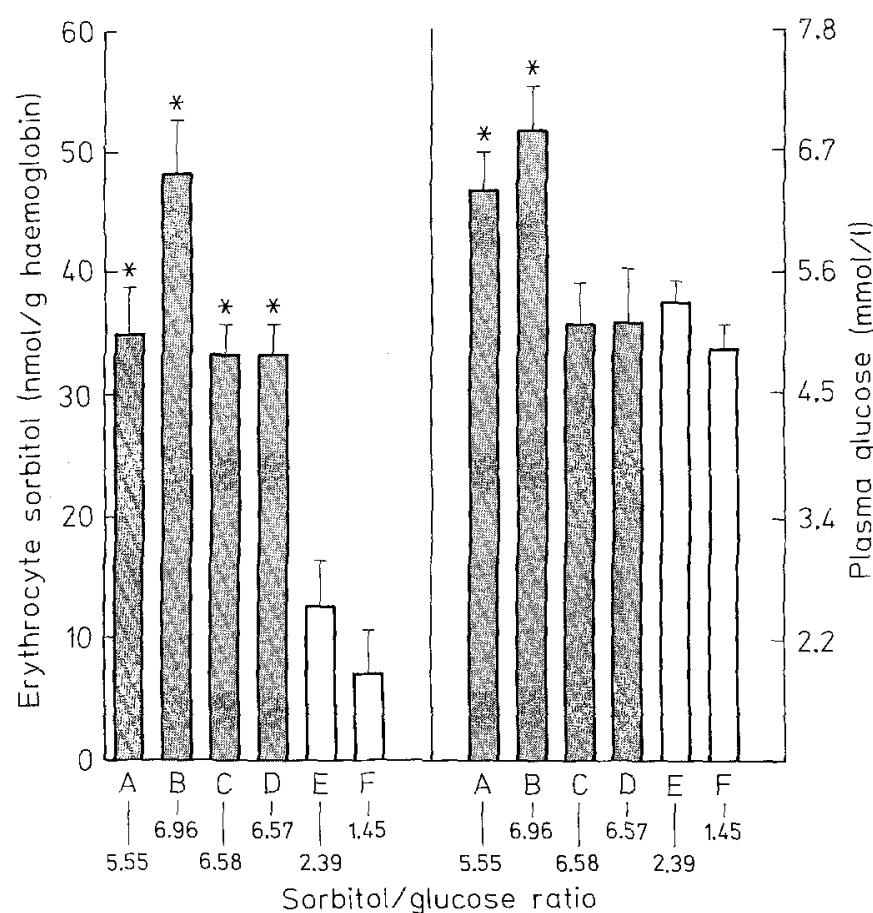

Fig.4. Erythrocyte sorbitol and plasma glucose levels were measured hourly in four Type 1 diabetic patients (番) and two control subjects $(\square)$ receiving intravenous insulin to maintain a plasma glucose $<$ $8.3 \mathrm{mmol} / \mathrm{l}$. The mean \pm SEM erythrocyte sorbitol levels were measured at hourly intervals during $12-14 \mathrm{~h}$ when the plasma glucose concentration was maintained $<8.33 \mathrm{mmol} / 1$. The sorbitol levels in erythrocytes from Type 1 diabetic patients were greater than control values. The coincident plasma glucose concentrations of two individuals were higher and two were the same as the non-diabetic control subjects. ${ }^{*} p<0.005$

for each determination on samples collected from the Type 1 diabetic patients were significantly greater than those from normal subjects (Table 1). The mean sorbitol/glucose ratio of 32 Type 1 diabetic subjects, who had fasting plasma glucose concentrations <
$5.6 \mathrm{mmol} / \mathrm{l}$, was $5.44 \pm 0.38$ (Fig. 1). This ratio is significantly greater than that of normal subjects $(p<0.001)$. To determine the influence of near-normal glucose concentrations upon sorbitol accumulation in vivo in Type 1 diabetic cells, the sorbitol/glucose values for 34 Type 1 diabetic subjects with coincident normal $\mathrm{HbA}_{1 \mathrm{c}}$ levels $(\leqslant 7 \%)$ were compared with the ratios measured in 50 normal individuals (Fig. 1). The mean sorbitol/ glucose ratio of erythrocytes from well controlled diabetic patients was greater than that found in normal erythrocytes $(p<0.001)$.

The influence of relatively stable extracellular glucose concentrations upon intracellular sorbitol accumulation is shown in Figure 2. Glucose consumption throughout each of the 3 -h incubation periods was similar to that previously reported $[11,12]$, indicating erythrocyte viability. Following $1 \mathrm{~h}$ of stabilization in the incubation medium, sorbitol accumulation in $2 \mathrm{~h}$ was greater in erythrocytes collected from diabetic than in control subjects (Fig. 2). When erythrocytes from diabetic subjects, with mean plasma glucose concentrations of $15.8 \pm 3.1 \mathrm{mmol} / \mathrm{l}$, were placed in a buffer containing $5.6 \mathrm{mmol} / 1$ glucose, an anticipated decrease in erythrocyte sorbitol occurred during the first hour of incubation. However, this decrease did not continue during the second hour, and erythrocyte sorbitol content returned to and exceeded the initial level at the conclusion of the 3-h incubation period. The mean sorbitol/ glucose ratio, using the erythrocyte sorbitol content and medium glucose concentration at the end of the 3-h incubation period, was greater in erythrocytes from Type 1 diabetic subjects $(7.7 \pm 1.04)$ than in control cells subjected to the same incubation system $(3.9 \pm 0.75$, $p<0.025$ ).

Erythrocyte fructose was measurable in 25 of the 63 incubation studies performed. These particular incubations were of erythrocytes from diabetic patients exposed to the highest glucose concentrations (16.7 and $27.8 \mathrm{mmol} / \mathrm{l}$ ) with the exception of three incubated in glucose $(5.6 \mathrm{mmol} / \mathrm{l})$. Erythrocyte fructose accumulation increased in response to the coincident erythrocyte sorbitol levels (Fig. 3). It is of interest that in two instances erythrocytes with modestly elevated sorbitol concentrations had fructose levels higher than the majority of erythrocytes with higher sorbitol concentrations. Fructose concentration also increased in the incubation media and correlated with the media glucose concentration at the completion of the incubation experiment $(r=0.67, p<0.001)$. Since the media did not contain measureable fructose before the erythrocyte incubation, the fructose measured at the end of the incubation is assumed to result from extracellular diffusion.

A study in vivo was performed to ensure erythrocyte viability during extended exposure to near-normal extracellular glucose concentrations. The erythrocyte sorbitol and plasma glucose levels were stable in the control subjects in spite of the ingestion of two meals during the $8 \mathrm{~h}$ of observation. In contrast, the erythro- 
cyte sorbitol and plasma glucose concentrations were variable in the four Type 1 diabetic patients monitored for $12-14 \mathrm{~h}$, as suggested by the larger standard error (Fig. 4). The erythrocyte sorbitol and sorbitol/glucose ratio in cells from the diabetic subjects did not return to normal levels in spite of 12-14 h of normal plasma glucose (Fig. 4).

\section{Discussion}

Sorbitol may be an aetiological factor in many of the complications of diabetes. Sorbitol levels in two tissues (lens and nerve) that seem to be damaged by elevated sorbitol correlate well with erythrocyte sorbitol concentrations [9].

Erythrocytes, as well as the target tissues of sorbitol toxicity, are freely permeable to glucose [12]. Therefore, activity of the polyol pathway is influenced by both aldose reductase activity and extracellular glucose concentration.

The sorbitol/glucose ratio was applied to standardize the sorbitol content of cells exposed to the wide range of plasma glucose concentrations characteristic of Type 1 diabetes. The finding of elevated sorbitol/glucose ratios in Type 1 diabetic individuals with normal plasma glucose and/or $\mathrm{HbA}_{1 \mathrm{c}}$ levels suggests that polyol pathway activity per unit of available glucose is greater in Type 1 diabetic than in normal subjects. The incubation of erythrocytes collected from Type 1 diabetic subjects showed increased sorbitol accumulation when compared with cells from non-diabetic controls incubated in an identical fashion.

The enhanced capacity of erythrocytes from Type 1 diabetic individuals to accumulate sorbitol was also associated with increased fructose accumulation. Fructose concentration in the incubation media, which was not measureable before incubation, correlated with the media glucose concentration after a 3-h incubation period. This observation of increased extracellular fructose, in addition to the report of Morrison et al. [11] showing media fructose increasing concomitantly with intracellular fructose during similar incubations, indicates that the intracellular accumulation of fructose is not the result of a block in fructose diffusion across the erythrocyte membrane.

Although increased intracellular fructose may inhibit sorbitol dehydrogenase activity, the immediate response of erythrocytes from Type 1 diabetic patients to reduced extracellular glucose $(5.6 \mathrm{mmol} / 1)$ was a coincident decrease in erythrocyte sorbitol. Since reduced substrate availability is more likely than reduced intracellular fructose in this situation, this suggests that impaired sorbitol oxidation, in response to product (fructose) inhibition, is not the cause of sorbitol accumulation in these cells. The modestly elevated sorbitol levels noted in two incubations in association with high intracellular fructose during the study lends further support for this observation.
Sorbitol/glucose ratios were measured in erythrocytes collected from a group of Type 1 diabetic patients taking Sorbinil (Pfizer, Groton, Connecticut, USA), an aldose reductase inhibitor [9]. The ratios before treatment $(2.9 \pm 0.12)$ were reduced to normal $(1.3 \pm 0.16)$, suggesting that inhibition of aldose reductase will correct the enhanced sorbitol accumulation noted in these cells. This response to an aldose reductase inhibitor, plus the evidence against impaired sorbitol oxidation, suggest that aldose reductive activity is increased in erythrocytes of Type 1 diabetic subjects.

A study in vivo was performed to evaluate the influence of normalized extracellular glucose for an interval greater than $3 \mathrm{~h}$ upon sorbitol content of cells from Type 1 diabetic subjects. The sorbitol content of those cells did not return to normal after 11-14 h of near-normal plasma glucose concentrations (Fig. 4). The sorbitol/glucose ratio for the Type 1 diabetic subjects in each case was greater than that observed for the normal subjects, and did return to normal after 11-14 h of normal glucose concentrations.

Normal individuals demonstrated stable (within the normal fasting range) hourly erythrocyte sorbitol and plasma glucose values in spite of the ingestion of two meals during the study. In contrast, wide fluctuations of sorbitol content occurred in erythrocytes from Type 1 diabetic patients in response to changes in extracellular glucose, as reported by others [14], revealing the acute responsiveness of this metabolic pathway to changes in extracellular glucose concentration. These observations suggest that, in addition to having altered sorbitol accumulation, erythrocytes from Type 1 diabetic subjects are more responsive to fluctuations of extracellular glucose than normal cells.

This alteration in polyol pathway activity appears to be acquired. One family (consisting of a mother and five children) is included in the patients reported. Two of the children have Type 1 diabetes. One of the affected children has an unaffected identical twin as supported by physical features and HLA typing. Both Type 1 diabetic children have elevated erythrocyte sorbitol and sorbitol/glucose ratios, while sorbitol in cells from unaffected family members are well within the normal range. Gabbay [15] has reported that peripheral nerves from experimental diabetic rats show greater sorbitol levels after incubation at high glucose concentration that do similar nerves from normal rats. Thus spontaneous diabetes, as well as experimental hyperglycaemia, seems to induce altered accumulation of sorbitol in tissues. The time required for induction of this enhanced activity and its return to normal have not been elucidated by these studies. It appears, however, that it requires more than $3 \mathrm{~h}$ for induction of increased activity and more than $12 \mathrm{~h}$ of normal plasma glucose for a return to the normal state. A recent report indicates that sorbitol accumulation in the erythrocyte correlates significantly with coincident sorbitol levels in the lens and nerve [9]. If the altered erythrocyte polyol pathway ac- 
tivity reflects altered activity in other tissues, then erythrocyte sorbitol levels and possibly sorbitol/glucose ratios may provide information helpful for selecting patients at greater risk for diabetic complications. Although the majority of Type 1 diabetic subjects reported had a sorbitol/glucose ratio above the upper range recorded for normal subjects, others had sorbitol/glucose ratios within the normal range. The variability in expression of altered polyol pathway activity in the Type 1 diabetic population studied may be related to the wellrecognized range of patient susceptibility to diabetic complications.

Acknowledgement. This study was supported in part by a grant funded under an agreement with the Health Program Office, Department of Health and Rehabilitative Services, State of Florida.

\section{References}

1. Chylack LT, Jr, Kinoshita JH (1969) A biochemical evaluation of a cataract induced in high glucose medium. Invest Opthalmol 8: $401-412$

2. Gabbay KH (1973) Role of sorbitol pathway in neuropathy. Adv Metab Disord (Suppl) 2: 417-424

3. Morrison AD, Clements RS, Jr, Winegrad AI (1972) Effects of elevated glucose concentration on the metabolism of the aortic wall. Clin Invest 51: 3114-3123

4. Malone JI, Knox G, Benford S, Tedesco TA (1980) Red cell sorbitol an indicator of diabetic control. Diabetes 29: 861-864

5. Gabbay KH, Cathcart ES (1974) Purification and immunologic identification of aldose reductase. Diabetes 23: 460 468

6. Beutler E, Guinto E (1974) The reduction of glyceraldehyde by human erythrocytes. J Clin Invest 53: 1258-1264
7. Clements RS, Jr, Morrison AD, Winegrad AI (1969) Polyol pathway in aorta: regulation by hormones. Science 1966: 1007-1008

8. Crabbe MHC, Halder AB (1980) Affinity chromatography of bovine lens aldose reductase, and a comparison of some kinetic properties of the enzyme from lens and human erythrocyte. Biochem Soc Trans 8: 194-195

9. Malone JI, Leavengood H, Peterson MJ, O'Brien MM, Page MG, Just LJ, Aldinger CE (1984) Red blood cell sorbitol: as an indicator of polyol pathway activity. Inhibition by sorbinil in insulin dependent diabetic subjects. Diabetes 33: 45-49

10. Sweeley CC, Bentley R, Makita M, Wells WW (1963) Gas-liquid chromatography of trimethylsilyl derivatives of sugars and related substances. J Am Chem Soc 85: 2497-2507

11. Morrison AD, Clements RS, Jr, Travis SB, Oski F, Winegrad AI (1970) Glucose utilization by the polyol pathway in human erythrocytes. Biochem Biophys Res Comm 40: 199-205

12. Travis SF, Morrison AD, Clements RS, Jr, Winegrad AI, Oski F (1971) Metabolic alterations in the human erythrocyte produced by increases in glucose concentration. J Clin Invest 50: 2104-2112

13. Cole RA, Soeldner JS, Dunne PJ, Bunn HF (1978) A rapid method for determination of glycosylated haemoglobin using high pressure liquid chromatography. Metabolism 27:289-301

14. Somers G, Depoorter I, Sener A, Malaisse WJ (1982) Sorbitol concentration in the plasma and erythrocytes of diabetic subjects. Diabetes Care 5: 319-321

15. Gabbay KH (1982) The sorbitol pathway and the complications of diabetes. New Engl Med 288: 831-836

Received: 25 July 1983

and in revised form: 23 August 1984

Dr. John I Malone

College of Medicine

University of South Florida

12901 North 30 Street

Box 15

Tampa, FL 33612

USA 